\title{
Influence of boric acid on radial structure of oxidized polyacrylonitrile fibers
}

https://doi.org/10.1515/epoly-2019-0010

Received July 27, 2018; accepted August 22, 2018.

\begin{abstract}
The surface modification of polyacrylonitrile (PAN) fibers with boric acid was utilized to modulate the homogeneity of the radial structure of the PAN fibers during thermal stabilization. Exothermic peaks of the fibers were put off by boric acid, and unreacted nitrile groups of the oxidized PAN fibers increased with the boron content, indicating that boric acid on the fiber surface had an retardant effect on the thermal stabilization of PAN fibers. The relative skin thicknesses of the oxidized PAN fibers were quantitatively measured by sulfuric acid etching and SEM observation. The value increased obviously with the boron content, which could be further elevated by increasing stabilization time or decreasing stabilization temperature. Oxidized PAN fibers with more homogeneous radial structure can thus be obtained with the modification of boric acid, which might be beneficial for the preparation of high performance carbon fibers.
\end{abstract}

Keywords: polyacrylonitrile fibers; boric acid; thermal stabilization; radial structure; carbon fibers

\footnotetext{
* Corresponding author: Xue-Fei Wang and Yong-Gang Zhang, National Engineering Laboratory for Carbon Fiber Preparation, Ningbo Institute of Material Technology \& Engineering, Chinese Academy of Sciences, Ningbo 315201, e-mail: wangxf@nimte. ac.cn (Xue-Fei Wang); zhangyonggang@nimte.ac.cn (Yong-Gang Zhang).

Xiao-Long Zhu, Ningbo Institute of Measurement and Testing, Ningbo 315048.

Chi Jiang, Guangzhou Institute of Chemistry, Chinese Academy of Sciences, Guangzhou 510650; University of Chinese Academy of Sciences, Beijing 100049.

Jian-Min Guan, Xin Qian, Shu-Lin Song and De-Hong Li, National Engineering Laboratory for Carbon Fiber Preparation, Ningbo Institute of Material Technology \& Engineering, Chinese Academy of Sciences, Ningbo 315201
}

\section{Introduction}

Polyacrylonitrile (PAN) based carbon fibers are widely used as important reinforcement materials in various areas like aircrafts, automobiles, construction reinforcement, sports good, etc., owning to their excellent properties like high strength, high modulus, ablation resistance, and low density (1-8). Thermal stabilization of PAN precursors is an important process in carbon fiber production, which converts linear polymers of PAN to ladder polymers. The fibers can then be resistant to melt in following carbonization process in an inert atmosphere.

A series of reactions including cyclization, dehydrogenation, oxidation, cross-linking, and decomposition happen during the stabilization process. On the account of that cyclization reaction is considered to be a reaction-controlled process $(9,10)$, raising temperature of heat treatment may be helpful to decrease retention time, and then improves the productivity. However, the oxidation reaction is thought to be controlled by the diffusion of oxygen (9). The formation of cyclized structures of the PAN chains is a prerequisite to oxidation, and the ladder structures could be attacked by oxygen as soon as they are formed $(10,11)$. The oxidation reaction of the polymers happens on the fiber surface is earlier than that in the inner core of the fiber. A dense skin of the fiber is formed. Oxygen diffuses gradually from the surface to the inner core of the fiber in the stabilization process, and the oxidation reaction happens simultaneously. The oxidized fiber thus owns inhomogeneous structure in radial direction, which is skin-core structure. The radial structure can be inherited by carbon fiber, which may have an impact on the mechanical properties of carbon fiber (12-14).

In order to improve the homogeneity of the cross section structure of the oxidized fiber, various reagents were introduced into the fiber or on the surface of the fiber before stabilization process. Metal compounds were commonly used. $\mathrm{KMnO}_{4}$ was chemically impregnated into polyacrylonitrile precursors and $\mathrm{MnO}_{4}-\mathrm{CN}$ conjugation 
was produced, which acted as a plasticizer and helped to catalyze cyclization reaction (15). The rate of stabilization was accelerated and the retention time was reduced. The cross section of the fibers was examined by optical microscope, and the cross section structure was homogenized by the modification. Similar results could also be obtained in other research (16). Oxygen in PAN fiber was thought to be an active site, and its content increased with the modification of $\mathrm{KMnO}_{4}$, which could promote the rate of cyclization and preoxidation. The concentrated oxygen could be found in modified PAN fiber, resulting in oxidized fiber with higher skincore ratio, which demonstrated that the homogeneous structure in the inner and outer parts of oxidized fiber could be improved (16). Carbon fiber developed from the $\mathrm{KMnO}_{4}$ modified PAN fiber showed homogenization of the cross section structure, which was observed by SEM (17). The tensile strength of the carbon fiber prepared from the chemically modified sample increased to 4.2 GPa, while that obtained from original PAN sample was $2 \mathrm{GPa}(18)$.

$\mathrm{CoSO}_{4}$ could also be used as a catalyst in the formation of ladder structures, reducing the temperature of cyclization. The resultant carbon fiber obtained from modified PAN fiber showed an improvement in the tensile properties (19). Cross section of oxidized fiber developed from treated PAN fiber showed homogeneous structure from SEM images, without large granulars or holes (19). Other metal compounds including Ni(II), Fe(II), Fe(III), $\mathrm{Zn}$ (II), $\mathrm{Cu}(\mathrm{I})$ (20-25), reagents such as hydrogen peroxide (26) and sulfur (27), and radiation of PAN with UV $(28,29)$, electron beam $(30,31)$, or gamma-rays $(32,33)$ were also introduced to modify PAN precursor fibers.

Boron may be another reagent to modulate the cross section structure of the fiber, putting off the initial temperature and peak temperatures and reducing the stabilization extent of the PAN fiber. PAN films doped with boric acid were obtained by adding boric acid into PAN solution. Ouyang et al. found that the initial temperature of cyclization increased from 193 to $224^{\circ} \mathrm{C}$ when PAN was treated with boric acid (34). As the content of boric acid increased, the suppression effect of boric acid on cyclization reaction was enhanced according to FTIR data (35). Although boric acid could retard the ionic cyclization, it had little influence on the radical cyclization. Boric acid could also be doped into PAN fiber prior to drying in the fiber spinning process. DSC profiles of the PAN fibers showed that boric acid inhibited the cyclization reaction weakly and hindered the oxidation reaction significantly, which was due to the inhibition effect of boric acid on the diffusion of oxygen (36).
Boric acid was coated on the fiber surface by soaking the PAN fibers in boric acid solution. Wu et al. considered boric acid acted as a cross-linking agent to tie the adjacent PAN molecular chains together, which could decrease the thermal shrinkage of the treated fiber. The cross-linked structures were thought to be less dense, and the diffusion of oxygen into the core region should be much easier (37). A sheath-core structure was noticed in the oxidized fibers without boric acid modification by optical microscope, which became a hollow tube in carbon fiber. Comparatively, structural difference between the sheath and core was alleviated in fiber coated with boric acid, and the sheath-core effect was not observed in carbon fiber by SEM (37). Besides, boron could catalyze the graphitization of carbon fibers, which was manifested as lowering the heating temperature during the graphitization process, promoting the increasement of the tensile modulus, and alleviating the decrease of the tensile strength (38-40).

Previous work mainly focuses on the retardant effect of boric acid on the chemical structure of PAN fiber in the thermal stabilization process. The influence of boric acid on the skin-core structure of the PAN fiber is less concerned. Primarily, the skin-core structure should be measured quantitatively. Instead of observation with optical microscope, the oxidized PAN fiber was firstly treated with concentrated sulfuric acid, which was learned from the treatment of fibers with aqueous sulfuric acid at reflux (9). The core was etched by sulfuric acid and the skin region remained. The skin thickness and the core diameter were then recorded by SEM observation, and the relative skin thickness (RST) was obtained (41). RST grew proportionally to the square root of the oxidation time $\left(\mathrm{t}^{1 / 2}\right)$ at 250 or $260^{\circ} \mathrm{C}$, and the oxidation reaction was considered to be controlled by the diffusive transport of oxygen $(9,41)$, which indicated that sulfuric acid etching and SEM observation method could be used to evaluate the skin-core structure of oxidized PAN fibers.

In this work, boric acid was loaded on the surface of the PAN fiber by immersing the fiber in the boric acid solution. PAN fibers with varing boron content were then thermally treated at a constant temperature. Sulfuric acid etching and SEM observation method was used to measure the skin thicknesses of the oxidized PAN fibers. It could help to understand the effect of boric acid on the radical structure of the oxidized PAN fibers. The mechanical properties, exotherms, and the preoxidation extents of the PAN fibers were also monitored. 


\section{Results and discussion}

\subsection{Physical properties of PAN monofilaments}

Boric acid modification was carried out in water bath at $80^{\circ} \mathrm{C}$. Even though the fiber was fixed in a constant length during the treatment process, the temperature or the boric acid might have an effect on the fiber structure. The physical properties of three PAN fibers were followed, PAN fiber, PAN fiber treated with $80^{\circ} \mathrm{C}$ water, and PAN fiber modified with boric acid. The major mechanical properties of the PAN monofilaments are listed in Table 1. These three kind of fibers have comparable tenacity and tensile modulus, indicating that boric acid modification or hot water treatment has little effect on the physical properties of the PAN fibers.

The tenacity of PAN fiber used is $6.58 \mathrm{cN} / \mathrm{dtex}$. This value is commonly found in PAN precursor fibers for carbon fiber preparation. The tensile strength of PAN fibers with different molecular weight was between $6.3 \sim 7.4 \mathrm{cN} / \mathrm{dtex}$ (42). The tenacity of PAN fiber obtained by wet-spinning with a postdrawing reached $6.08 \mathrm{cN} / \mathrm{dtex}$ (43). The precursor fibers for modification with hydrogen peroxide had a tenacity higher than $6.5 \mathrm{cN} / \mathrm{dtex}$ and a tensile modulus higher than $105 \mathrm{cN} / \mathrm{dtex}$ (26). Comparatively, the PAN fiber used in this work has a higher tensile modulus.

\subsection{Thermal behavior of PAN fibers in air}

Figure 1 shows the DSC curves of PAN fibers with different boron content at a heating rate of $10^{\circ} \mathrm{C} / \mathrm{min}$ under air atmosphere. All the samples have two obvious exothermic peaks, one peak is at around $270^{\circ} \mathrm{C}$ and the other one is at around $330^{\circ} \mathrm{C}$. The former one is attributed to cyclization reaction, dehydrogenation reaction and oxidation reaction, and the latter one to certain intermolecular cross-linking reaction and aromatization of structure (44-46). Detailed parameters from DSC curves are listed in Table 2. When PAN fiber is modified with boric acid,

Table 1: The physical properties of PAN monofilaments.

\begin{tabular}{lrrrr}
\hline Samples & $\begin{array}{r}\text { Fineness } \\
\text { dtex }\end{array}$ & $\begin{array}{r}\text { Tenacity } \\
\text { cN/dtex }\end{array}$ & $\begin{array}{r}\text { Tensile modulus } \\
\text { cN/dtex }\end{array}$ & $\begin{array}{r}\text { Elongation } \\
\%\end{array}$ \\
\hline PAN & $0.77 \pm 0.02$ & $6.58 \pm 0.60$ & $131.17 \pm 21.07$ & $12.29 \pm 0.72$ \\
PAN $^{\star a}$ & $0.81 \pm 0.03$ & $6.26 \pm 0.40$ & $130.98 \pm 19.12$ & $13.01 \pm 0.78$ \\
PAN-B0.4 & $0.76 \pm 0.02$ & $6.85 \pm 0.60$ & $124.69 \pm 21.13$ & $12.99 \pm 0.69$ \\
\hline
\end{tabular}

${ }^{\text {aPAN }}{ }^{*}$ was the fiber immersed in deionized water at $80^{\circ} \mathrm{C}$ for $5 \mathrm{~min}$ without boric acid.

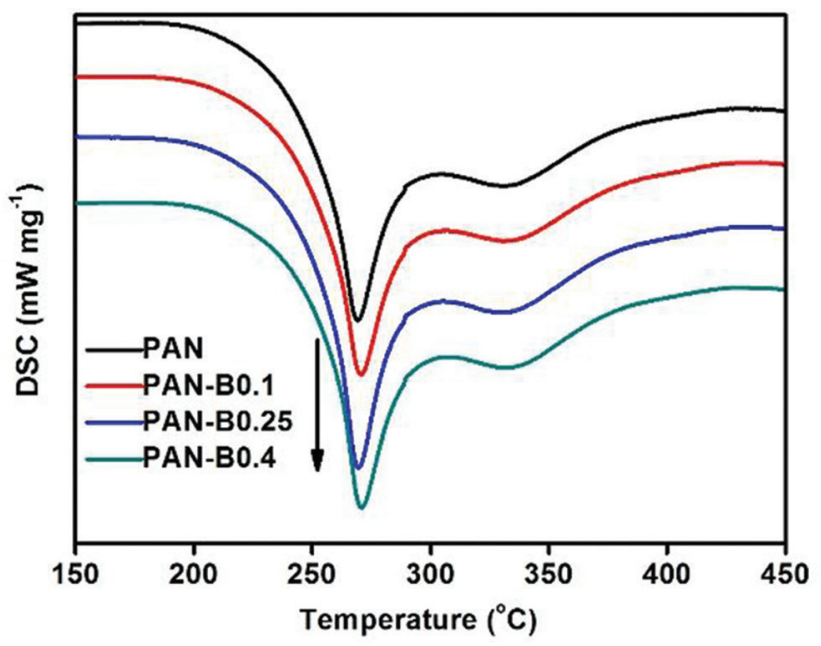

Figure 1: DSC curves of PAN fibers with different boron content in air at $10^{\circ} \mathrm{C} / \mathrm{min}$.

Table 2: DSC parameters of PAN fibers with different boron content in air.

\begin{tabular}{lrrrrrr}
\hline Samples & $T_{i}\left({ }^{\circ} \mathrm{C}\right)$ & $T_{f}\left({ }^{\circ} \mathrm{C}\right)$ & $\Delta T\left({ }^{\circ} \mathrm{C}\right)$ & $T_{p 1}\left({ }^{\circ} \mathrm{C}\right)$ & $T_{p 2}\left({ }^{\circ} \mathrm{C}\right)$ & $\Delta H\left(\mathrm{~J} \mathrm{~g}^{-1}\right)$ \\
\hline PAN & 189.7 & 442.4 & 252.7 & 269.1 & 331.0 & 1972 \\
PAN-B0.1 & 187.8 & 440.5 & 252.7 & 270.6 & 331.1 & 1990 \\
PAN-B0.25 & 190.7 & 439.1 & 248.4 & 269.7 & 330.8 & 2103 \\
PAN-B0.4 & 191.3 & 443.1 & 251.8 & 270.8 & 331.9 & 1980 \\
\hline
\end{tabular}

$T_{p 1}$ increases from 269.1 to $270.8^{\circ} \mathrm{C}$ and $T_{p 2}$ from 331.0 to $331.9^{\circ} \mathrm{C}$. Meanwhile, $T_{i}$ shifts to a higher temperature, but $\Delta H$ changes little. According to the DSC data, boric acid shows a weak retardant effect on the cyclization reaction and oxidation reaction of PAN fiber.

\subsection{Chemical composition of oxidized fibers}

Figure 2a shows a typical spectrum of original PAN fiber without thermal treatment. There are three characteristic peaks, one at $2240 \mathrm{~cm}^{-1}$ due to the stretching vibration of $\mathrm{C} \equiv \mathrm{N}$ bond $\left(\mathrm{v}_{\mathrm{C} \equiv \mathrm{N}}\right)$, one at $1454 \mathrm{~cm}^{-1}$ due to strong $\mathrm{C}-\mathrm{H}$ in-plane deformation vibration of $-\mathrm{CH}_{2}$ groups $\left(\delta_{\mathrm{C}-\mathrm{H}}\right)$, and another one at $2940 \mathrm{~cm}^{-1}$ due to $\mathrm{C}-\mathrm{H}$ stretching vibration of $-\mathrm{CH}_{2}$ groups $\left(\mathrm{v}_{\mathrm{CH}}\right)$. There are also two additional peaks at $1730 \mathrm{~cm}^{-1}$ and $1260 \mathrm{~cm}^{-1}$, ascribed to stretching vibration of $\mathrm{C}=\mathrm{O}$ or $\mathrm{C}-\mathrm{O}$, which is due to the presence of the comonomer. After thermal treatment at $260^{\circ} \mathrm{C}$ for $10 \mathrm{~min}$, all the characteristic peaks of PAN fiber decreases, as shown in Figure $2 \mathrm{~b}$. At the same time, one peak at $1585 \mathrm{~cm}^{-1}$ can be observed. The peak is ascribed to the products of the reaction of nitrile groups, which is considered to be $\mathrm{C}=\mathrm{C}$ and $\mathrm{C}=\mathrm{N}$ (44). 


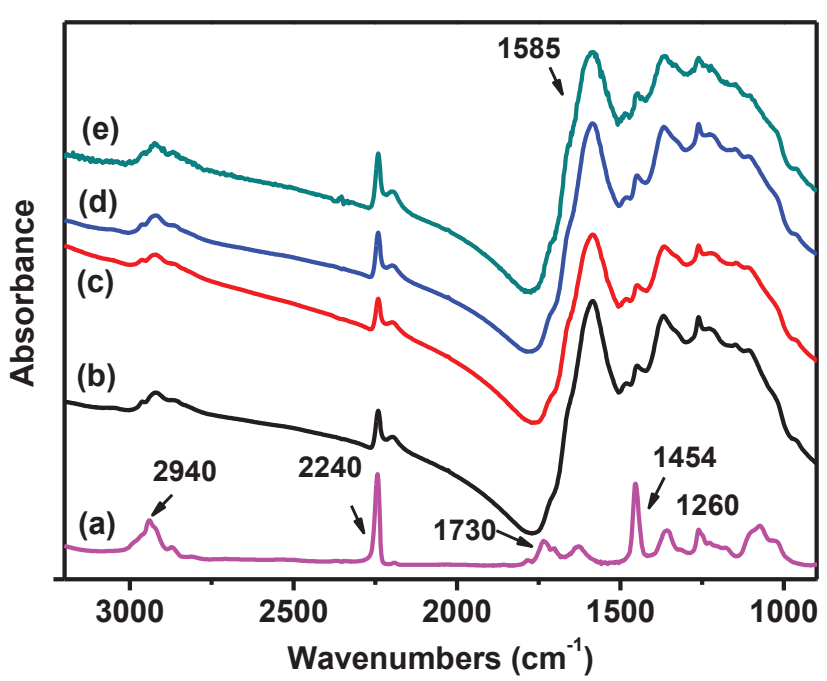

Figure 2: FTIR spectra of original PAN fiber (a) and fibers with varing boron content treated at $260^{\circ} \mathrm{C}$ (b) 0 , (c) $0.1 \%$ (d) $0.25 \%$, and (e) $0.4 \%$.

With the increase of boric acid on the surface of PAN fibers, the decrease of absorbance at $2240 \mathrm{~cm}^{-1}$ is reduced (Figures 2c-e). It means that fewer nitrile groups are involved in the stabilization process. $f$ is used to determine the fraction of unreacted nitrile groups according to the expression proposed by Collins et al. (47).

$$
f=\frac{A B S\left(2240 \mathrm{~cm}^{-1}\right)}{A B S\left(2240 \mathrm{~cm}^{-1}\right)+0.29 \times A B S\left(1585 \mathrm{~cm}^{-1}\right)}
$$

Where $f$ is the fraction of unreacted nitrile groups and ABS is the absorbance intensity.

The $f$ values of oxidized PAN fibers are shown in Figure 3. The $f$ value of PAN fiber without boric acid modification is 0.50 , and it increases to 0.51 when the fiber surface is coated with $0.1 \%$ boron. With the increasing of boron content, the $f$ value increases, which means that there are more unreacted nitrile groups remaining in the fiber. The $f$ value increases to 0.62 for oxidized PANB0.4 fiber. Boric acid on the fiber surface thus retards the stabilization process of PAN obviously. The retardation effect of boric acid was also observed by FTIR when boric acid was added to PAN film (35).

The mechanism of ion initiated cyclization reaction showed that the carboxylic groups of itaconic acid comonomer initiated the cyclization reaction as a nucleophilic group (48). The PAN fiber here is modified with boric acid, which is a Lewis acid that can accept a pair of electron. In the initiation process, the electron pair on carboxylic group may be captured by boron, and hydroxyl oxygen loses the ability to transfer the free electron pair to nitrile groups. Boric acid thus inhibits the cyclization process, which can then happen at higher temperature

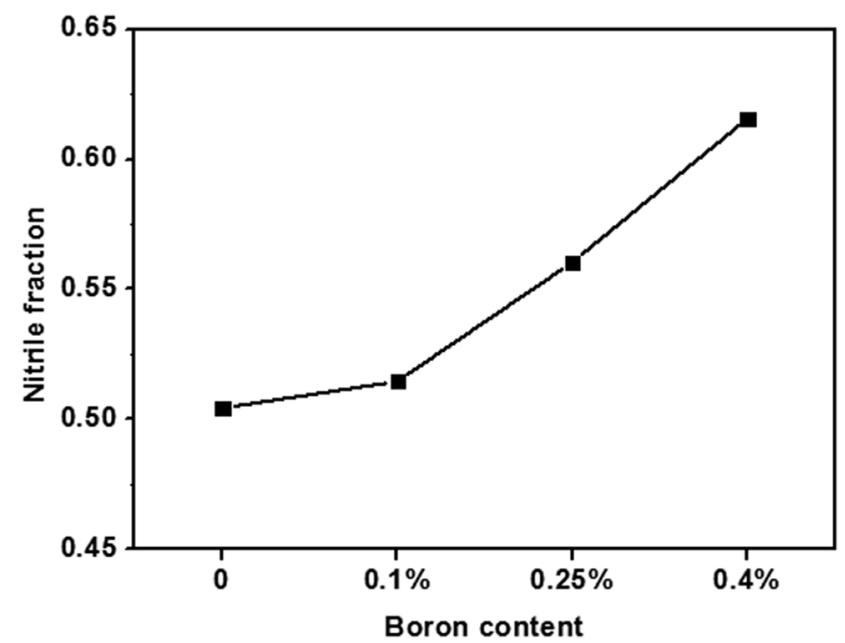

Figure 3: The fraction of unreacted nitrile groups of PAN fibers with varing boron content after thermal treatment at $260^{\circ} \mathrm{C}$ for $10 \mathrm{~min}$.

by radical initiation. Therefore, after thermal treatment, the boric acid modified PAN has more unreacted nitrile groups than that of unmodified one, and unreacted nitrile groups are elevated with the boron content. Grassie et al. also mentioned that boric acid played a role as inhibitor of the reaction rather than as an acid initiator, which was indicated by DTA and TG (25).

\subsection{The radial structure of oxidized PAN fibers}

According to previous experiments, skin part and core part of oxidized PAN fibers can be easily differentiated by stabilization at $260^{\circ} \mathrm{C}(41) .260^{\circ} \mathrm{C}$ was chosen as the stabilization temperature in the work. SEM images of cross sections of boric acid coated PAN fibers after stabilization and following sulfuric acid etching are shown in Figure 4. The retention time of stabilization is $10 \mathrm{~min}$ (Figures 4a-d) and $60 \mathrm{~min}$ (Figures 4a'-d'), respectively. Figure 4a shows cross section of PAN fiber after stabilization at $260^{\circ} \mathrm{C}$ for $10 \mathrm{~min}$ and following sulfuric acid etching. The inner core of the fiber is eroded by sulfuric acid and a hollow tube is observed. Keeping the retention time of stabilization for $10 \mathrm{~min}$, the core diameters of the modified PAN fibers decrease with the boron content on the fiber surface (Figures $4 \mathrm{c}$ and $4 \mathrm{~d}$ ). When the retention time of stabilization is prolonged, the core diameters of the four oxidized fibers can be reduced evidently (Figures 4a'-d').

The relative skin thicknesses (RST) of the oxidized fibers are shown in Figure 5. The thickness of the fibers increases obviously with the boron content, from 0.62 of original PAN fiber to 0.69 of PAN-B0.4 fiber. Thicker skin can be obtained in fiber with higher boron content. 

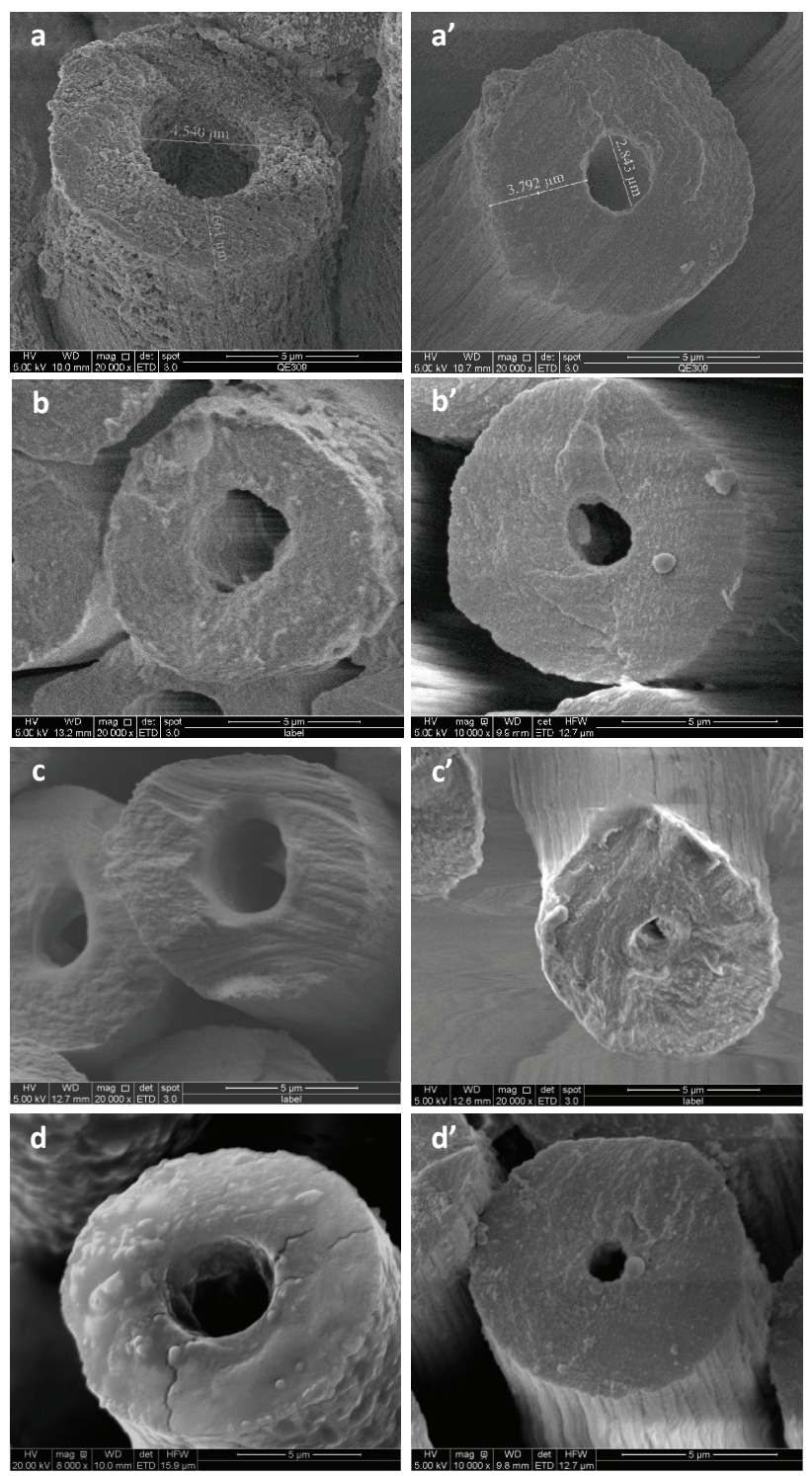

Figure 4: The cross section images of PAN fibers following stabilization at $260^{\circ} \mathrm{C}$ for $10 \mathrm{~min}(\mathrm{a}-\mathrm{d})$ and $60 \mathrm{~min}$ (a'-d') and sulfuric acid etching: (a, a') PAN, (b, b') PAN-B0.1, (c, c') PAN-B0.25, (d, d') PAN-B0.4.

More homogeneous structure of the fiber can also be obtained by increasing the retention time of thestabilization process, offering more time for the diffusion of oxygen $(49,50)$. When the retention time increases from $10 \mathrm{~min}$ to $60 \mathrm{~min}$, the core diameter of the PAN fiber decreases and the RST increases to 0.75 . Similar phenomenon also happens to other modified PAN fibers, and increasing the retention time of the stabilization process improves the homogeneous structure of the fibers. Moreover, with the increasing of the boron content, the core diameter of the fibers is reduced and the RST of the fibers increases gradually. When PAN-B0.4 fiber is stabilized at $260^{\circ} \mathrm{C}$ for $60 \mathrm{~min}$, the RST of the oxidized fiber reaches 0.87 .

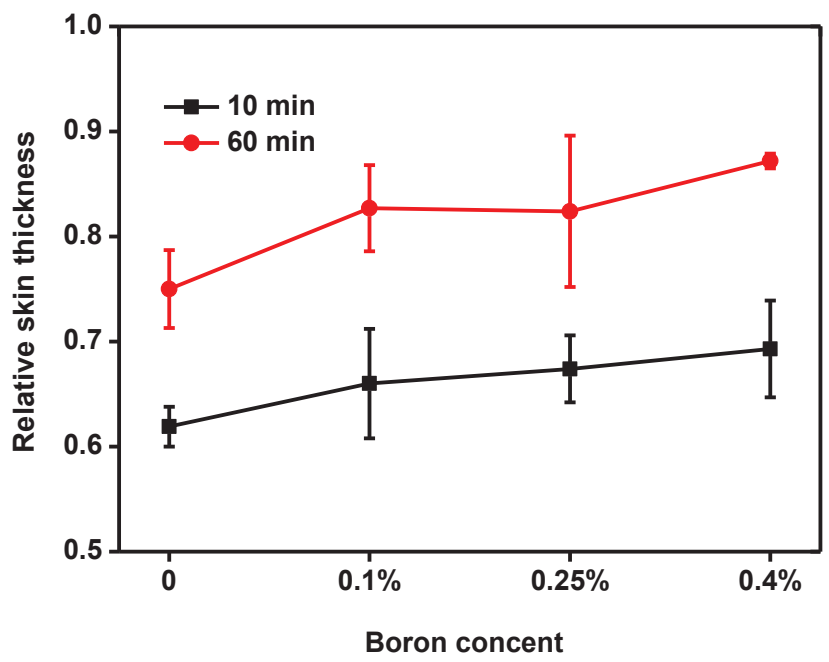

Figure 5: The relative skin thickness of PAN fibers with varing boron content oxidized at $260^{\circ} \mathrm{C}$ for $10 \mathrm{~min}$ and $60 \mathrm{~min}$.
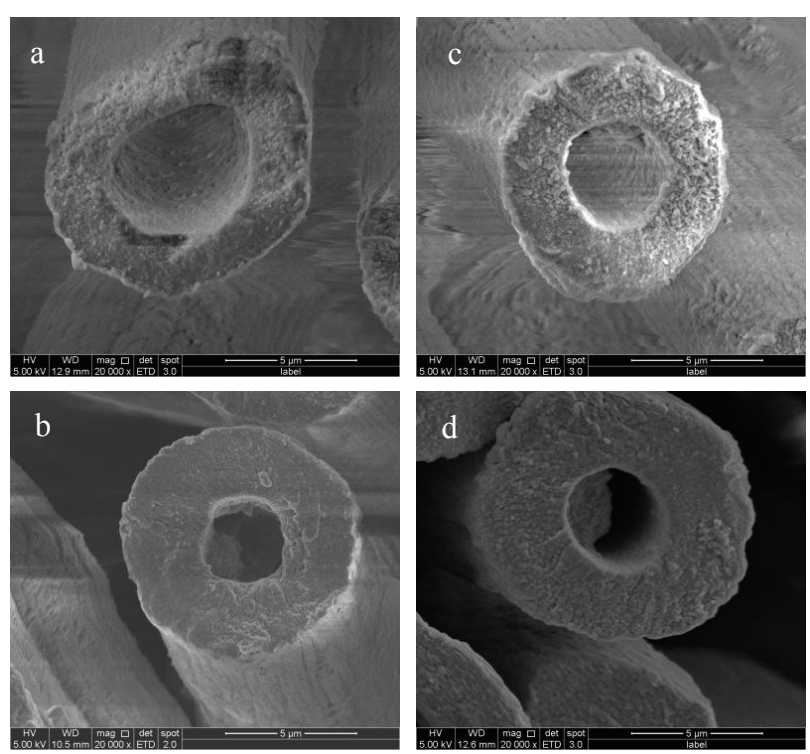

Figure 6: PAN fiber (a, c) and PAN-B0.4 (b, d) stabilized at $270^{\circ} \mathrm{C}$ $(a, b)$ and $280^{\circ} \mathrm{C}(c, d)$ after sulfuric acid etching.

When retention time is kept at $10 \mathrm{~min}$, raising the temperature of the thermal treatment results in more inhomogeneous structure of the fibers. As for PAN fiber, when the temperature increases to $270^{\circ} \mathrm{C}$, core diameter increases, and the skin thickness decreases, which is shown in Figure 6a. When the temperature is further elevated to $280^{\circ} \mathrm{C}$, the skin-core structure changes little (Figure $6 \mathrm{~b}$ ). Compared with oxidized PAN fiber, the oxidized fiber prepared from PAN-B0.4 fiber has a significantly reduced core (Figures 6c and 6d). Boric acid on the surface of the fiber can improve the homogeneous structure of the oxidized fibers.

Figure 7 shows that the relative skin thicknesses of the oxidized fibers of PAN and PAN-B0.4 change with 


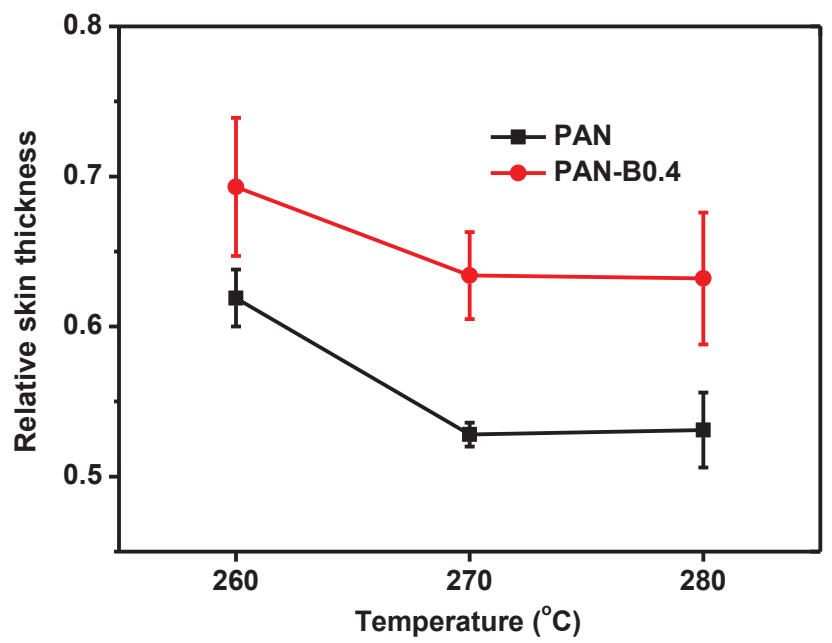

Figure 7: The relative skin thickness of PAN fiber and PAN-B0.4 fiber stabilized at different temperatures for $10 \mathrm{~min}$.

the stabilization temperature. When the stabilization temperature increases from $260^{\circ} \mathrm{C}$ to $270^{\circ} \mathrm{C}$, the RST decreases to 0.53 , and the value changes little when the temperature further increases to $280^{\circ} \mathrm{C}$. For PAN-B0.4 sample, theRSTdecreaseswith thestabilization temperature and the value is 0.63 for the fiber stabilized at $270^{\circ} \mathrm{C}$, which is comparable with that for the fiber oxidized at $280^{\circ} \mathrm{C}$.

In the stabilization process, boric acid probably reacted with hydroxyl groups, acting as a kind of cross-linking agent for the polymer chains (34). The increase of the binding energy of boron in $\mathrm{B}-\mathrm{O}$ bonding was considered to be assigned to $B_{1 s}$ in boronate, further verifying the cross-linking reactions between boric acid and hydroxyl groups (37). Research on the $\mathrm{B}_{2} \mathrm{O}_{3}$ /polymer films indicated that the improvement on the thermal stability of the composite films may be attributed to the inhibition of physical blockage of active sites for oxidation probably by boron oxide (51). Boric acid decomposes into metaboric acid at around $170^{\circ} \mathrm{C}$ and is completely converted to boron oxide at around $350^{\circ} \mathrm{C}$. In this work, boron oxide may exist on the surface of the boric acid modified fibers when the stabilization temperature is up to $280^{\circ} \mathrm{C}$. The inhibition of physical blockage of boron oxide and the binding of B-O may retard the oxidation reaction of PAN, resulting the improvememt of homogeneity of the radial structure of the PAN fibers.

Sulfuric acid etching can thus be used to differentiate the structure of oxidized PAN fiber with different cross section structure. The method was used to treat partially stabilized PAN fiber with different co-polymer composition, fiber $\mathrm{C}$ and fiber $\mathrm{M}$, which had the same diameters. After etching in $50 \mathrm{wt} \%$ aqueous sulfuric acid at reflux for $24 \mathrm{~h}$, the former presented a hollow tube with inner core eroded and the latter remained intact (9). In our experiment, concentrated sulfuric acid is introduced to reduce the treatment temperature to room temperature and also the etching time to $5 \mathrm{~min}$ (41). The skin core structure of the oxidized PAN fibers can be controlled by temperature and retention time in stabilization process. In constant-temperature heat treatment process, higher core ratio of fibers could be obtained with less retention time or higher temperature. The skin thickness of the fiber grew proportionally to the square root of the oxidation time, which indicated that the oxidation reaction was controlled by the diffusive transport of oxygen (41). In this experiment, boric acid can be used to modulate the skin core structure of the oxidized PAN fibers.

\section{Conclusions}

Polyacrylonitrile (PAN) fibers was coated with boric acid by immersing the fiber in boric acid solution. Boric acid modification had little effect on the physical properties of the PAN fibers, indicated by the comparable tensile properties of PAN and PAN-B0.4 fibers. DSC curves showed that boric acid could put off the exothermic peaks, and the reagent could retard the cyclization and oxidation reactions. After thermal stabilization at $260^{\circ} \mathrm{C}$, the nitrile groups of the original PAN fiber decreased and the value of the fraction of unreacted nitrile groups increased with boron content. The relative skin thickness of the fiber increased with boron content, which can be further elevated with the retention time of the stabilization whatever the boron content was. When the stabilization temperature decreased from $280^{\circ} \mathrm{C}$ to $260^{\circ} \mathrm{C}$, the relative skin thickness of the fiber increased, which was further raised in the fiber modified with $0.4 \%$ boron. Boric acid can thus be introduced onto the PAN fibers surface to improve the homogeneity of the radial structure of PAN fibers during the thermal stabilization.

\section{Experimental}

\subsection{Materials}

The polyacrylonitrile (PAN) fibers, containing 1 wt $\%$ itaconic acid as comonomer, were prepared by Ningbo Institute of Material Technology \& Engineering, Chinese Academy of Sciences (CAS). Boric acid and sulfuric acid in analytical grade were obtained from Sinopharm Chemical Reagent Co., Ltd., China.

Fiber samples with different boron contents were obtained by immersing the PAN fibers in boric acid solutions 
Table 3: Fiber samples with boric acid modification.

\begin{tabular}{lrr}
\hline Samples & $\begin{array}{r}\text { Content of Boron } \\
\text { (wt\%) }\end{array}$ & $\begin{array}{r}\text { Concentration of boric acid } \\
\text { solution (wt\%) }\end{array}$ \\
\hline PAN & 0 & 0 \\
PAN-B0.1 & 0.1 & 8 \\
PAN-B0.25 & 0.25 & 10 \\
PAN-B0.4 & 0.4 & 12 \\
\hline
\end{tabular}

with different concentrations at $80^{\circ} \mathrm{C}$ for $5 \mathrm{~min}$. These fibers were fixed on a frame to avoid shrinkage of the fibers in the dipping process, and then dried in an oven at $100^{\circ} \mathrm{C}$ for $2 \mathrm{~h}$.

Around $0.5 \mathrm{~g}$ boric acid-modified fiber was pretreated by adding the fiber in $50 \mathrm{~mL}$ nitric acid solution ( $5 \mathrm{wt} \%)$ for $48 \mathrm{~h}$. The acidified solution was then analyzed by inductively coupled plasma-atomic emission spectroscopy (ICP-AES, Perkin-Elmer Optima 2100) and the boron content on the fiber surface was obtained. The fiber samples and the boron contents on the fiber surface were shown in Table 3.

\subsection{Thermal stabilization of PAN fibers}

The thermal stabilization process of the PAN fibers was carried out in air in an oven. After treatment at a certain temperature for a given time, the fibers were taken out for further test.

\subsection{Characterization}

The physical properties of PAN monofilaments were monitored on the XQ-1 Fiber Tensile Tester and XD-1 Vibration Fiber Fineness Tester (Shanghai New Fiber Instrument Co., Ltd., China). Tensile rate was $20 \mathrm{~mm} / \mathrm{min}$, and gauge length was $20 \mathrm{~mm}$. The quoted result for each fiber was an average of values of 50 measurements.

The heat release behavior of the fiber samples in air was followed by using DSC (Netzsch STA 449 F3). The measurements were conducted from room temperature to $450^{\circ} \mathrm{C}$ with a heating rate of $10^{\circ} \mathrm{C} / \mathrm{min}$. The parameters of initial temperatures $\left(T_{i}\right)$, final temperature $\left(T_{f}\right)$, their difference $(\Delta T)$, peak temperature $\left(T_{p}\right)$ and the change of enthalpy $(\Delta H)$ of the DSC exotherms were listed.

The chemical composition of the fibers after thermal treatment was monitored by FTIR (Thermo Nicolet 6700). $\mathrm{KBr}$ disk was made of fiber powder and $\mathrm{KBr}$. The infrared spectra were recorded with a resolution of $4 \mathrm{~cm}^{-1}$ in the range of 4000-400 $\mathrm{cm}^{-1}$ for 32 times.

Oxidized PAN fibers were firstly solidified in collodion for $30 \mathrm{~min}$, then were cut to make the cross section exposed. The cross sections of the fibers were exposed to 95-98\% sulfuric acid for $5 \mathrm{~min}$. The samples were taken out, then washed with deionized water for $1 \mathrm{~min}$, and dried at $105^{\circ} \mathrm{C}$ for $2 \mathrm{~h}$. The cross section images of the oxidized PAN fiber after the treatment of sulfuric acid were then monitored by Field Emission Scanning Electron Microscope (FEI Quanta FEG 250, USA). The diameter of the fiber $\left(D_{f}\right)$ and that of the core $\left(D_{c}\right)$ were then analyzed by Image-pro Plus. The relative skin thickness (RST) of the oxidized fiber was evaluated as $R S T=\left(D_{f}-D_{c}\right) / D_{f}$. The result was an average of values of five fiber samples.

Acknowledgements: Financial support from National Natural Science Foundation of China (No. 51503216) and Zhejiang Provincial Natural Science Foundation of China (Nos. LQ16E030003 and LY18E080037) are gratefully acknowledged.

\section{References}

1. Frank E., Hermanutz F., Buchmeiser M.R., Carbon Fibers: Precursors, Manufacturing, and Properties. Macromol Mater Eng, 2012, 297, 493-501.

2. Liu Y.D., Kumar S., Recent Progress in Fabrication, Structure, and Properties of Carbon Fibers. Polym Rev, 2012, 52, 234-258.

3. Hiremath N., Mays J., Bhat G., Recent Developments in Carbon Fibers and Carbon Nanotube-Based Fibers: A Review. Polym Rev, 2017, 57, 339-368.

4. Cai X., Zhang C.Q., Zhang S.S., Fang Y.P., Zou D.C., Application of carbon fibers to flexible, miniaturized wire/fiber-shaped energy conversion and storage devices. J Mater Chem A, 2017, 5, 2444-2459.

5. Newcomb B.A., Processing, structure, and properties of carbon fibers. Compos Part A-Appl S, 2016, 91, 262-282.

6. Frank E., Steudle L.M., Ingildeev D., Sporl J.M., Buchmeiser M.R., Carbon Fibers: Precursor Systems, Processing, Structure, and Properties. Angewe Chem Int Edit, 2014, 53, 5262-5298.

7. Duan G.G., Zhang H., Jiang S.H., Xie M.Y., Peng X.W., Chen S.L., et al., Modification of precursor polymer using co-polymerization: A good way to high performance electrospun carbon nanofiber bundles. Mater Lett, 2014, 122, 178-181.

8. Duan G.G., Fang H., Huang C.B., Jiang S.H., Hou H.Q., Microstructures and mechanical properties of aligned electrospun carbon nanofibers from binary composites of polyacrylonitrile and polyamic acid. J. Mater. Sci., 2018, 53, 15096-15106.

9. Warner S.B., Peebles L.H., Uhlmann D.R., Oxidative Stabilization of Acrylic Fibers .1. Oxygen-Uptake and General-Model. J Mater Sci, 1979, 14, 556-564.

10. Xiao S.J., Cao W.Y., Wang B., Xu L.H., Chen B.H., Mechanism and Kinetics of Oxidation During the Thermal Stabilization of Polyacrylonitrile Fibers. J Appl Polym Sci, 2013, 127, 3198-3203.

11. Watt W., Johnson W., Mechanism of Oxidization of Polyacrylonitrile Fibers. Nature, 1975, 257, 210-212.

12. Mittal J., Bahl O.P., Mathur R.B., Single step carbonization and graphitization of highly stabilized PAN fibers. Carbon, 1997, 35, 1196-1197. 
13. Li D.H., Lu C.X., Wu G.P., Yang Y., An F., Feng Z.H., et al., Structural heterogeneity and its influence on the tensile fracture of PAN-based carbon fibers. Rsc Adv, 2014, 4, 60648-60651.

14. Zhou G.S., Liu Y.Q., He L.L., Guo Q.G., Ye H.Q., Microstructure difference between core and skin of T700 carbon fibers in heat-treated carbon/carbon composites. Carbon, 2011, 49, 2883-2892.

15. Liu J., Zhang W.X., Structural changes during the thermal stabilization of modified and original polyacrylonitrile precursors. J Appl Polym Sci, 2005, 97, 2047-2053.

16. Wang Y., Yin W.Y., In: Xiong Y. (Ed.), Proceeding of the Fourth International Conference on Surface and Interface Science and Engineering (Lanzhou, China), 2011, 18.

17. Zhang W.X., Jie L., Effect of post-spinning modification on the PAN precursors and resulting carbon fibers. J Wuhan Univ Technol, 2006, 21, 44-48.

18. Mathur R.B., Mittal J., Bahl O.P., Sandle N.K., Characteristics of $\mathrm{KMnO}_{4}$-Modified Pan Fibers - Its Influence on the Resulting Carbon-Fibers Properties. Carbon, 1994, 32, 71-77.

19. Zhang W.X., Wang Y.Z., Manufacture of carbon fibers from polyacrylonitrile precursors treated with $\mathrm{CoSO}_{4}$. J Appl Polym Sci, 2002, $85,153-158$

20. Ko T.H., Huang L.C., Preparation of High-Performance CarbonFibers from Pan Fibers Modified with Cobaltous Chloride. J Mater Sci, 1992, 27, 2429-2436.

21. Morawski A.W., Ueda M., Inagaki M., Preparation of transition metal-carbon material from polyacrylonitrile incorporated with inorganic salts. J Mater Sci, 1997, 32, 789-795.

22. Zhang W.X., Wang Y.Z., Wang Y.X., Cai H.S., Li M.S., Effect of $\mathrm{NiSO}_{4}$ on the structure and properties of PAN precursors and resultant carbon fibres. Acta Polym Sin, 2001, 670-673.

23. Karacan I., Erdogan G., Ferric Chloride Assisted Thermal Stabilization of Polyacrylonitrile Precursor Fibers Prior to Carbonization. J Inorg Organomet P, 2012, 22, 1016-1027.

24. Mathur R.B., Bahl O.P., Kundra K.D., Characterization of Modified Pan Precursors. J Mater Sci Lett, 1986, 5, 757-759.

25. Grassie N., Mcguchan R., Pyrolysis of Polyacrylonitrile and Related Polymers .4. Thermal Analsis of Polyacrylonitrile in Presence of Additives. Eur Polym J, 1971, 7, 1503-\&.

26. Liu L.Q., Chen H.F., Pan D., Modification of Polyacrylonitrile Precursor Fiber with Hydrogen Peroxide. Fiber Polym, 2012, 13, 587-592.

27. Wang Y.X., Wang C.G., Effect of adding sulphur to the dope on properties of modified polyacrylonitrile fibers. J Appl Polym Sci, 2007, 105, 3774-3779.

28. Yuan H.W., Wang Y.S., Yu H.W., Wei Z., Ge B., Mei Y.J., Effect of UV Irradiation on PAN Precursor Fibers and Stabilization Process. J Wuhan Univ Technol, 2011, 26, 449-454.

29. Paiva M.C., Kotasthane P., Edie D.D., Ogale A.A., UV stabilization route for melt-processible PAN-based carbon fibers. Carbon, 2003, 41, 1399-1409.

30. Shin H.K., Jeun J.P., Kang P.H., The Characterization of Polyacrylonitrile Fibers Stabilized by Electron Beam Irradiation. Fiber Polym, 2012, 13, 724-728.

31. Park S., Yoo S.H., Kang H.R., Jo S.M., Joh H.I., Lee S., Comprehensive stabilization mechanism of electron-beam irradiated polyacrylonitrile fibers to shorten the conventional thermal treatment. Sci Rep-UK, 2016, 6,

32. Zhao W.Z., Lu Y.G., Jiang J.Q., Hu L.Y., Zhou L.X., The effect of gamma-ray irradiation on the microstructure and thermal properties of polyacrylonitrile fibers. Rsc Adv, 2015, 5, 23508-23518.
33. Liu W.H., Wang M.H., Xing Z., Wu G.Z., The free radical species in polyacrylonitrile fibers induced by gamma-radiation and their decay behaviors. Radiat Phys Chem, 2012, 81, 835-839.

34. Ouyang Q., Wang H.J., Cheng L., Sun Y., Effect of boric acid on the stabilization of poly(acrylonitrile-co-itaconic acid). J Polym Res, 2007, 14, 497-503.

35. Ouyang Q., Cheng L., Wang H.J., Li K.X., FTIR Study of the retardation effect of boric acid on the cyclization reaction of polyacrylonitrile. e-Polymers, 2009.

36. Chen L., Lu C.X., Huang F., Li H., Liu Y.D., Lu Y.G., Doping boric acid into polyacrylonitrile fibers prior to drying process and the effects on stabilization. J Mater Sci, 2017, 52, 9452-9464.

37. Wu G.P., Lu C.X., Wang Y.Y., Ling L.C., Effect of Boric Acid on Oxidative Stabilization of Polyacrylonitrile Fibers. Fiber Polym, 2011, 12, 979-982.

38. Wang H., Xiao H., Lu Y.G., Jiang J.Q., The catalytic effect of boron nitride on the mechanical properties of polyacrylonitrile-based carbon fiber. J Mater Sci, 2016, 51, 10690-10700.

39. Wen Y., Lu Y.G., Xiao H., Qin X.Y., Further investigation on boric acid catalytic graphitization of polyacrylonitrile carbon fibers: Mechanism and mechanical properties. Mater Des, 2012, 36, 728-734.

40. Chen J.E., Xiong X., Xiao P., Zhang H.B., The catalytic effect of boric acid on polyacrylonitrile-based carbon fibers and the thermal conductivity of carbon/carbon composites produced from them. Carbon, 2010, 48, 2341-2346.

41. Wang X.F., Zhu X.L., Jiang C., Zhang Y.G., Yang J.X., Evaluation of skin-core structure of stabilized polyacrylonitrile fiber by sulfuric acid etching method. New Chem Mater, 2016, 44, 35-37, 40.

42. Wang Q.-F., Wang C.-G., Wang Y.-X., Yang M.-W., Yu M.-J., Effect of structure on tensile strength of PAN fiber. Gongneng Cailiao/J Funct Mater, 2006, 37, 1787-1789.

43. Kirsten M., Meinl J., Schonfeld K., Michaelis A., Cherif C., Characteristics of wet-spun and thermally treated poly acrylonitrile fibers. J Appl Polym. Sci, 2016, 133, 43698.

44. Mittal J., Bahl O.P., Mathur R.B., Sandle N.K., Ir Studies of Pan Fibers Thermally Stabilized at Elevated-Temperatures. Carbon, 1994, 32, 1133-1136.

45. Mathur R.B., Bahl O.P., Mittal J., A New Approach to Thermal Stabilization of Pan Fibers. Carbon, 1992, 30, 657-663.

46. Gupta A., Harrison I.R., New aspects in the oxidative stabilization of pan-based carbon fibers. Carbon, 1996, 34, 1427-1445.

47. Collins G.L., Thomas N.W., Williams G.E., Kinetic Relationships between Heat-Generation and Nitrile Consumption in the Reaction of Poly(Acrylonitrile) in Air at 265-Degrees-C. Carbon, 1988, 26, 671-679.

48. Tsai J.S., Lin C.H., Effect of Comonomer Composition on the Properties of Polyacrylonitrile Precursor and Resulting CarbonFiber. J Appl Polym Sci, 1991, 43, 679-685.

49. Karacan I., Erdogan G., The Role of Thermal Stabilization on the Structure and Mechanical Properties of Polyacrylonitrile Precursor Fibers. Fiber Polym, 2012, 13, 855-863.

50. Kong L.Q., Liu H., Cao W.Y., Xu L.H., PAN fiber diameter effect on the structure of PAN-based carbon fibers. Fiber Polym, 2014, 15, 2480-2488.

51. Jian S.J., Liu S.W., Chen L.L., Zhou S.Z., Fan P.C., Zeng Y., et al., Nano-boria reinforced polyimide composites with greatly enhanced thermal and mechanical properties via in-situ thermal conversion of boric acid. Compos Commun, 2017, 3, 14-17. 